\title{
CORNERSTONE 4
}

\section{AMPLIFYING IMPACT}

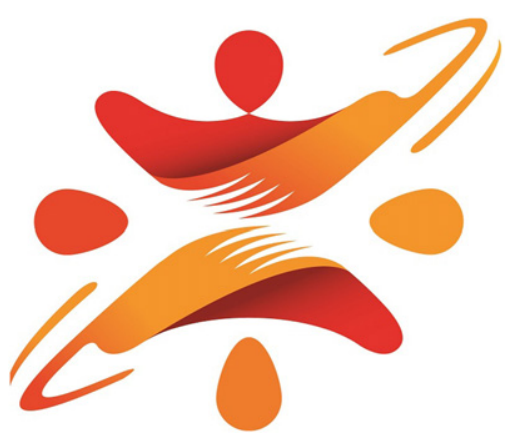

THE MULTIPLIER EFFECT: SUCCESS REQUIRES COLLECTIVE COMMITMENT

Catalytic behaviors work together to create improved outcomes. They synergistically amplify the impact that individuals and teams have on results. Whether in business, sports, or the arts, exceptional teams have an impactfirst approach. The impact may include financial results, points scored and wins tallied, or spectacular performances on stage.

The work associated with amplifying impact is embedded in the team and organizational stories that become the fabric of an organization's culture. How do we drive an uncompromising approach to excellence? Who successfully mentors and coaches our up-and-coming talent? What imaginative ideas emerge to drive innovation and ensure sustainable organizational success? 
If you are going to achieve excellence in big things, you develop the habit in little matters. Excellence is not an exception, it is a prevailing attitude.

- Colin Powell in The Leadership Secrets of

Colin Powell (2002)

We have addressed three cornerstones thus far: Building Credibility, Creating Cohesion, and Generating Momentum. Each includes three competencies that were described behaviorally and can be developed with focus and effort. Are these now well established in your tool set? If so, you are in a stronger position to amplify the impact that you have on the team whether or not you are in a position of formal authority.

Mastery of the competencies in this fourth cornerstone, listed below, will strengthen your ability to lead with steadiness and with urgency when necessary - especially when coupled with a commitment to driving performance.

- Pursues Excellence.

- Mentors and Coaches Others to Excel.

- Proposes Imaginative Solutions. 